\title{
Silicon grating structures for optical fiber interfacing and III-V/silicon opto-electronic components
}

\author{
Gunther Roelkens*a, $^{\text {, }}$, Diedrik Vermeulen ${ }^{c}$, Yanlu Li ${ }^{\mathrm{a}, \mathrm{b}}$, Muhammad Muneeb ${ }^{\mathrm{a}, \mathrm{b}}$, Nannicha \\ Hattasan $^{\mathrm{a}, \mathrm{b}}$, Eva Ryckeboer ${ }^{\mathrm{a}, \mathrm{b}}$, Yannick Deconinck ${ }^{\mathrm{a}, \mathrm{b}}$, Dries Van Thourhout ${ }^{\mathrm{a}, \mathrm{b}}$, Roel Baets ${ }^{\mathrm{a}, \mathrm{b}}$ \\ ${ }^{a}$ Photonics Research Group, INTEC Department, Ghent University-IMEC, Sint-Pietersnieuwstraat 41, 9000 \\ Ghent, Belgium \\ ${ }^{\mathrm{b}}$ Center for Nano- and Biophotonics (NB-Photonics), Ghent University, Belgium \\ ${ }^{c}$ currently with Acacia Communications, 3 Clock Tower Place, Suite 210, Maynard, MA 01754, USA
}

\begin{abstract}
In this paper, we review our work on efficient, broadband and polarization independent interfaces between a silicon-oninsulator photonic IC and a single-mode optical fiber based on grating structures. The high alignment tolerance and the fact that the optical fiber interface is out-of-plane provide opportunities for easy packaging and wafer-scale testing of the photonic IC. Next to fiber-chip interfaces we will discuss the use of silicon grating structures in III-V on silicon optoelectronic components such as integrated photodetectors and microlasers.
\end{abstract}

Keywords: high contrast grating, fiber-chip coupling, III-V/silicon opto-electronics

\section{INTRODUCTION}

Silicon photonics is emerging as a very attractive platform to integrate optical functions on a single chip. This has several reasons. First of all, CMOS fabrication tools and processes can be used to realize these devices, much in the same way, as silicon electronic ICs are fabricated. This well-developed technology infrastructure allows realizing low-cost photonic ICs, given the economy of scale and the high yield of the processes. In order to obtain compact photonic ICs, which further reduces the cost of the IC and at the same time also improves the performance of active optoelectronic devices, high refractive index contrast optical waveguide structures should be used, given the high confinement of the optical mode in such a waveguide system. Silicon-on-insulator (SOI) wafers, containing a silicon waveguide layer supported by a buried $\mathrm{SiO} 2$ layer on top of a silicon carrier wafer provide the opportunity to realize optical waveguides with very high omnidirectional refractive index contrast. This however brings along another challenge: how to efficiently couple light between a standard single mode fiber and the silicon photonic wires. High contrast diffraction gratings can provide an elegant solution here, on which we will elaborate in the next section. A second problem with the use of silicon photonics is how to monolithically integrated a coherent light source in the infrared, which is required for many applications. The heterogeneous integration of III-V semiconductors on silicon waveguide circuits is considered as an attractive solution to realize this goal. Again, high contrast grating structures in the silicon waveguide layer can be used to realize advanced III-V on silicon light sources and other opto-electronic components on a silicon platform, as will be discussed in this paper.

\section{DIFFRACTION GRATINGS FOR OPTICAL FIBER INTERFACING}

When considering the performance metrics of fiber-chip interfacing structures, one can identify the coupling efficiency of the interface, the optical bandwidth of the structure, its polarization independence, the compliance with standard single mode fiber and the associated alignment tolerance, the footprint of the coupling structure and the return loss of the interface. In the following we will outline several device designs and demonstrations that optimize a subset of these performance metrics. The considered grating structures are all second order grating structures, which, when excited from

*gunther.roelkens@intec.ugent.be; phone 0032(0)92648930 
the silicon waveguide, diffract light under a small angle (typically 10 degrees) with respect to the surface normal. This small detuning is chosen to substantially reduce the second order Bragg reflection in the silicon waveguide.

\subsection{High efficiency grating couplers}

A high-efficiency interface between a standard single-mode optical fiber and a silicon photonic IC is a prerequisite for using grating couplers in practical applications. The coupling efficiency is determined by two factors: first of all, there is the grating directionality $D$, defined as the ratio of the optical power diffracted toward the optical fiber to the total diffracted power. Second, there is the mismatch between the diffracted field profile and the Gaussian mode of the optical fiber, which results in additional losses. Indeed, in first order, the diffracted field profile originating from a uniform grating can be considered to be exponentially decaying, with a decay length inversely proportional to the grating strength. This implies that there exists an optimal grating strength for which the overlap with the Gaussian fiber mode is maximal. For a fiber-mode-field diameter of $10.4 \mu \mathrm{m}$ (as is the case for standard single mode fiber at $1550 \mathrm{~nm}$ ), this optimal field coupling strength is $0.14 / \mu \mathrm{m}$. For this optimal configuration, the excess loss due to the mode profile mismatch is about $-1 \mathrm{~dB}$. In this work we focus on maximizing the directionality of the grating. Three approaches were considered, all yielding similar coupling efficiencies to single mode fiber of about $-1.5 \mathrm{~dB}$. The approaches are outlined in Figure 1. Figure 1(a) and 1(b) illustrate the use of bottom mirrors [1,2] to redirect the downwards diffracted light towards the optical fiber. The distance between the grating and the mirror needs to be optimized to obtain the optimum coupling strength. In Figure 1(c) the grating structure is optimized such that it is intrinsically very directive, i.e. nearly no light is diffracted towards the $\mathrm{SiO}_{2}$ substrate [3]. SEM pictures of the fabricated structures are shown in Figure 2. While approach (a) was a proof of principle based on a few samples, approach (b) and (c) are demonstrated on 200mm SOI wafers and fabricated in a CMOS pilot line [4].

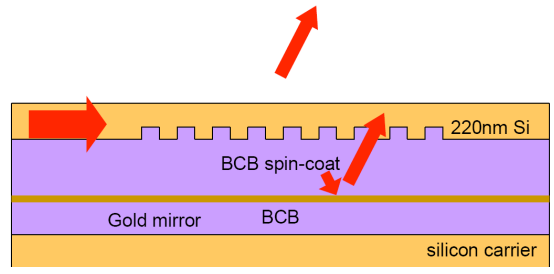

(a)

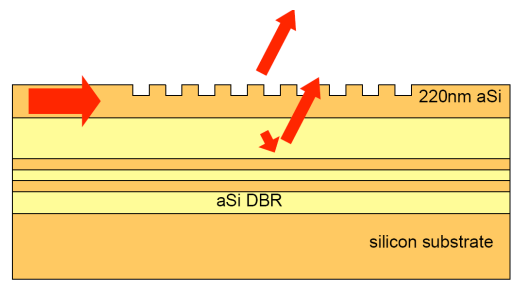

(b)

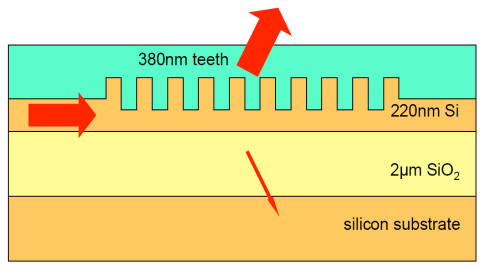

(c)

Figure 1: Strategies to improve the grating directionality: (a) using a bottom metal mirror to redirect the downwards diffracted light, (b) using a bottom DBR mirror and (c) making the grating intrinsically directional by defining a silicon overlay.

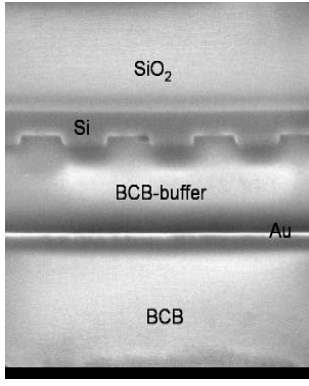

(a)

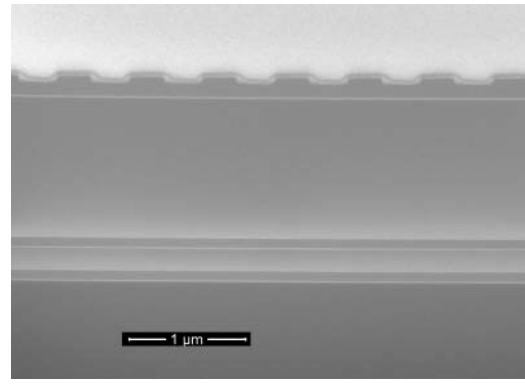

(b)

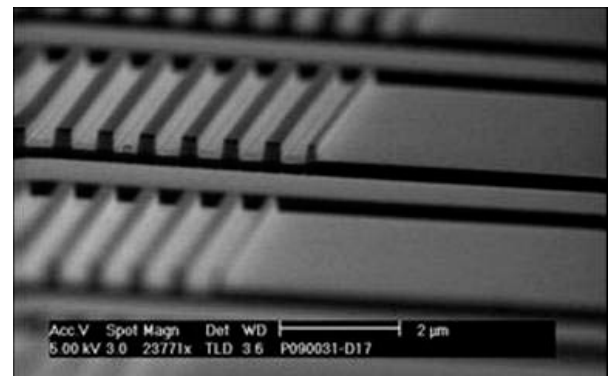

(c)

Figure 2: Scanning electron microscope images of fabricated high-efficiency grating coupler structures based on a metal bottom mirror, an amorphous silicon bottom mirror and a silicon overlay

\subsection{Large optical bandwidth grating couplers}

Since the diffraction properties of the grating structure are used to couple light between an optical fiber and the silicon waveguide circuit, the coupling efficiency is inherently wavelength dependent. However, since the considered gratings have a very short coupling length (in order to match the diffracted field size with that of the fiber mode), considerable 
optical bandwidth can still be obtained. This can be explained by considering the exponentially decaying diffracted field profile as a superposition of plane waves. The angular distribution of these plane waves will become broader when the grating is shorter (and hence, stronger if one assumes that all the light needs to be diffracted from the silicon waveguide over the grating length). This implies that a change in wavelength (implying a rotation of this angular distribution of plane waves) will have a lower impact when the grating is shorter, resulting in a larger optical bandwidth for shorter (stronger) gratings. The exact optical bandwidth depends on the specific design. For the considered high-efficiency grating couplers described in the previous section based on the silicon overlay grating, the $-1 \mathrm{~dB}(-3 \mathrm{~dB})$ excess loss is $45 \mathrm{~nm}(90 \mathrm{~nm})$ around a wavelength of $1.55 \mu \mathrm{m}$ [4]. While this optical bandwidth is sufficient for many applications, there are applications, which require a larger optical coupling bandwidth, especially because of the fact that light in two distinct (and widely separated) wavelength bands needs to be processed by the photonic IC. An important application satisfying this description is the use of integrated transceivers for fiber-to-the-home (FTTH) optical networks, in which at the subscriber side a 1310-nm wavelength is used to transmit upstream data over the network, while a 1490-nm downstream optical signal needs to be processed (and vice versa at the central office side). As the wavelength span required in this application exceeds the optical bandwidth of the fiber-to-waveguide grating coupler an alternative approach is required, which makes used of both the left and right entrance waveguide of a one-dimensional grating coupler, as shown in Figure 3. This allows the realization of fiber-chip coupling interfaces that work in two distinct wavelength bands, which can be tuned by changing the grating period and the fiber coupling angle [5].

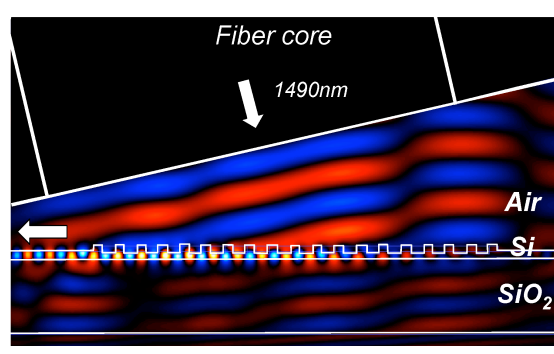

(a)

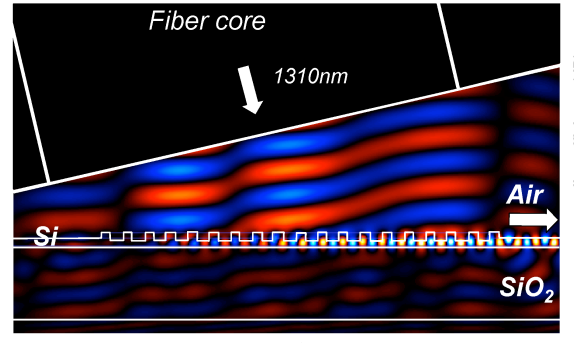

(b)

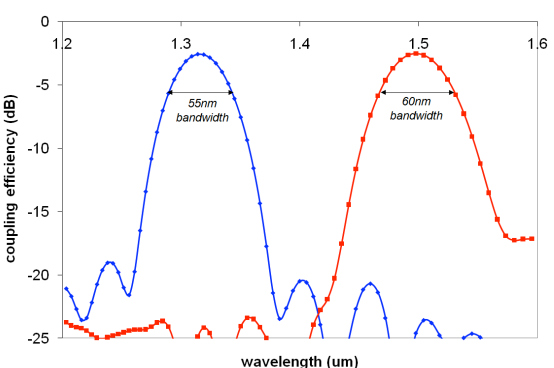

(c)

Figure 3: one-dimensional grating duplexer design operating in the 1.3 and 1.49 um wavelength range: (a) coupling of $1490 \mathrm{~nm}$ wavelength TE polarized light to the silicon waveguide circuit, (b) coupling of $1310 \mathrm{~nm}$ wavelength TE polarized light to the silicon waveguide circuit, and (c) coupling efficiency spectrum for the optimized structure.

\subsection{Polarization diversity grating couplers}

In many applications there is a need to have a photonic integrated circuits that behave in a polarization independent way. Typically, in high index contrast waveguide structures this is achieved using a polarization diversity approach. In this configuration, the two orthogonal polarizations in the optical fiber are both coupled to the photonic IC, but into a different waveguide. Thereby, the two polarizations are split on the chip and can be further processed by two parallel photonic ICs. This implies that polarization independent operation can be achieved, at the expense of doubling the size of the IC. Two approaches have been demonstrated to realize that goal: one is to use a two-dimensional grating structure that is surface illuminated and couples light to the same polarization mode in two nearly orthogonal waveguides [6]. In a second approach the two entrance waveguides to a one-dimensional grating coupler structure are used to couple the two orthogonal polarizations to the TE and TM mode of the respective waveguides [7]. An additional polarization rotator is then required to obtain identical polarizations in both arms. Both approaches are outlined in Figure 4. Since the 2D grating structure is illuminated from a fiber that is tilted about 10 degrees off normal, some residual polarization dependent loss remains, which can however be removed in a fiber-to-fiber configuration by implementing a 180 degree phase shift in one arm of the polarization diversity circuit [8]. This way polarization dependent losses below $1 \mathrm{~dB}$ can be obtained over a broad wavelength range. 


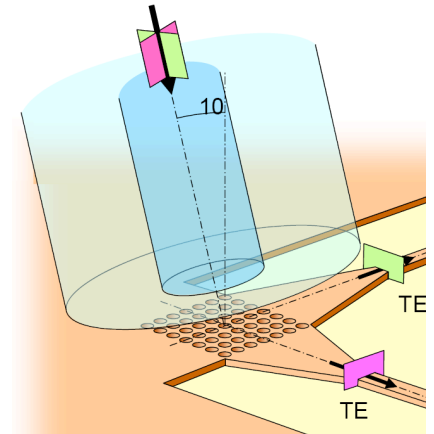

(a)

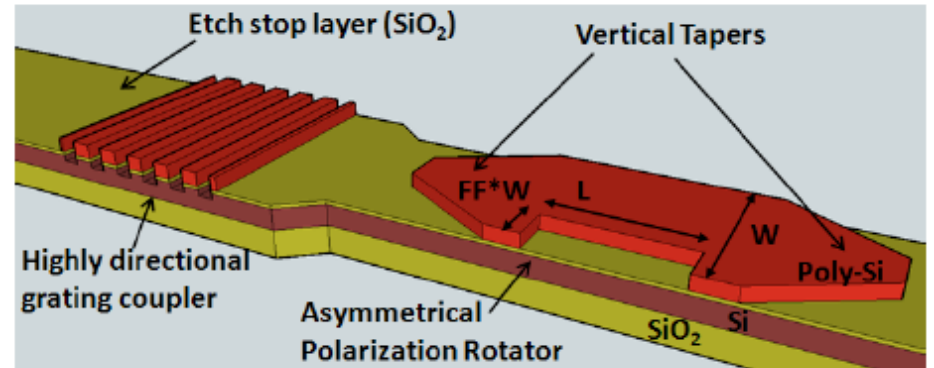

(b)

Figure 4: (a) 2D grating coupler approach and (b) 1D grating coupler approach in combination with a polarization rotator to realize polarization independent photonic integrated circuits.

\subsection{Focusing grating couplers}

The diffraction grating structures considered in this paper are very compact, since they only occupy the area of the core of a standard single-mode optical fiber, roughly $10 \mu \mathrm{m}$-by-10 $\mu \mathrm{m}$. However, in order to convert the 10 - $\mu \mathrm{m}$-wide optical waveguide mode to that of a $0.5-\mu \mathrm{m}$ single-mode waveguide, a relatively long adiabatic taper is required, on the order of $150 \mu \mathrm{m}$ in length. This makes the total device structure less compact, thereby increasing the overall footprint of the photonic IC. One elegant way to circumvent this issue is to use focusing grating structures, instead of the waveguide structures described earlier. A1-D grating structure can be made to focus the coupled beam on a micrometer size aperture by curving the grating lines, as shown in Figure 5(a) [9]. In this case, each grating line represents a part of an ellipse, while all ellipses describing the focusing grating have a common focal point, namely at the waveguide aperture. A similar design consideration can be made for 2-D grating couplers, by considering them as a "superposition" of two 1-D curved grating structures, as shown in Figure 5(b) [10].
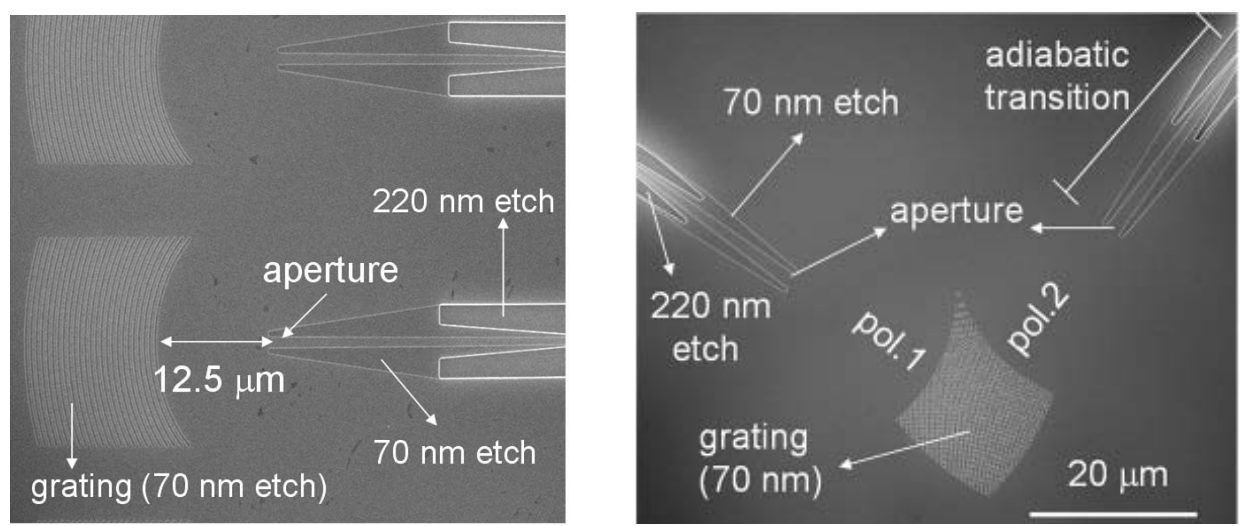

Figure 5: (a) 1D focusing grating coupler structure; (b) 2D focusing grating coupler structure

\subsection{Reflectionless grating couplers}

There are two main sources for back-reflection at the interface between a silicon waveguide and a diffraction grating. Once is the already mentioned second-order Bragg reflection, which can be relatively broadband for high-index contrast gratings. A second source of backreflection is the Fresnel reflection at the waveguide/grating coupler interface, which is very difficult to eliminate. By using off-axis focusing grating couplers, this reflection can be diverted away from the entrance waveguide as shown schematically in Figure 6 [11]. This way, very low back reflection grating couplers (-50dB return loss) were experimentally realized [12]. 


\subsection{Grating coupler structures for the short-wave infrared}

While all previous results were focused on the telecommunication wavelength range, there is a continuing trend to also explore the use of silicon photonic integrated circuits at longer wavelengths. The main rationale for this is to exploit twophoton absorption free efficient nonlinear processes in a silicon waveguides at wavelengths beyond $2.2 \mathrm{um}$ and also to use the silicon photonic platform as a spectroscopic sensing platform, to probe the infrared absorption fingerprint of different molecules. In both applications efficient fiber to chip grating coupler structures are required. Such grating structures, both scaled-up versions of the previously presented structures [13] and also sub-wavelength single etch grating structures were recently demonstrated for the short-wave infrared. We will elaborate on this at the conference.

\subsection{Wafer-scale testing of photonic integrated circuits}

One major advantage of the use of diffraction grating couplers to interface with a silicon photonic circuit is that there is no need for a cleaved and polished chip facet to interface with the optical fiber. This allows wafer-scale testing of the silicon photonic integrated circuits. In the Photonics Research Group we built a $200 \mathrm{~mm}$ wafer-scale optical prober setup that allows to automatically scan over the wafer and to probe the thousands of photonic components. This provides a very valuable tool for monitoring the uniformity and yield of the photonic integrated circuits.

\section{HIGH CONTRAST GRATINGS FOR III-V/SILICON OPTO-ELECTRONIC COMPONENTS}

\subsection{III-V on silicon photodetector integration}

While Ge photodetectors are currently being considered as the best approach towards integrated high-speed photodetectors, using silicon photonic integrated circuits for spectroscopic applications requires low dark current, high sensitivity devices realized in III-V semiconductors, especially when a very broad wavelength range needs to be addressed that goes beyond the cut-off wavelength of Ge. For this purpose heterogeneously integrated and surface illuminated III-V photodetectors can be used that use a diffraction grating to interface with the silicon photonic integrated circuit. Since the numerical aperture of the photodetector is very large, also the optical bandwidth associated with the diffraction coupler interface can cover several $100 \mathrm{~nm}$. Such integrated photodetector structures were recently demonstrated in the short-wave infrared wavelength range [14].

\subsection{III-V on silicon laser integration}

The holy grail for silicon photonics is the integrated laser source. Practical approaches nowadays rely on the heterogeneous integration of III-V semiconductors on top of the silicon waveguide circuits. In order to realize single wavelength lasers on this III-V on silicon platform, silicon gratings can be used. While we recently demonstrated distributed feedback lasers and distributed Bragg reflection lasers on this platform, we will elaborate here on the use of resonant grating mirrors to realize integrated microlaser sources on a silicon platform.

In order to reduce the power consumption and footprint of a laser, the overlap of the optical field with the III-V multiple quantum well region should be optimized and the laser cavity volume should be minimized. However, still the wavelength selective feedback is preferably implemented in the silicon waveguide layer, given the fact that high accuracy patterning is more easily achieved in silicon than in III-V layers. These requirements can be realized by defining a waveguide in the III-V layer, which improves the confinement in the active region. In order to couple the light to the silicon waveguide layer, typically an intra-cavity spot-size converter is used to squeeze the mode out of the III-V mesa into the silicon waveguide. In the silicon waveguide, wavelength selective feedback can be realized by using a combination of Bragg gratings and ring resonators, which can be thermo-optically tuned [15]. However, low-loss and low-back reflection tapers are difficult to realize in such a device geometry, which again impacts the threshold current and slope efficiency of the device. Recently, we proposed a novel approach for realizing low-power consumption, single mode lasers that are robust against mode hops based on resonant mirrors, as illustrated in Figure 6(a) [16]. Still the laser mode is confined to the III-V wire but instead of adiabatically tapering all the light from the III-V wire to the silicon layer towards the wavelength selective feedback structure, the III-V waveguide is now evanescently coupled to two silicon cavities, one at each facet of the III-V wire. The silicon cavities are identical and the coupling to the III-V waveguide provides high, narrow banded reflectivity back into that III-V waveguide as described in [16]. Figure 6(b) shows a typical reflection and transmission spectrum of a single silicon cavity coupled to a III-V waveguide. The reflectivity of this mirror is mainly determined by the quality of the silicon cavity. One can prove that if the losses in the cavity are sufficiently small, this mirror will reflect near $100 \%$ of the incident light back into the III-V waveguide. This 
is similar to an add-drop filter, which is always critically coupled if the coupling section is symmetric and the losses in the ring are sufficiently low. Typically, the silicon cavities are 20-30um long, and because they provide such high reflectivity, the laser gain section can also be short, on the order of a few 10's of micrometers. This means that the device length can be in the range of 50-100um.

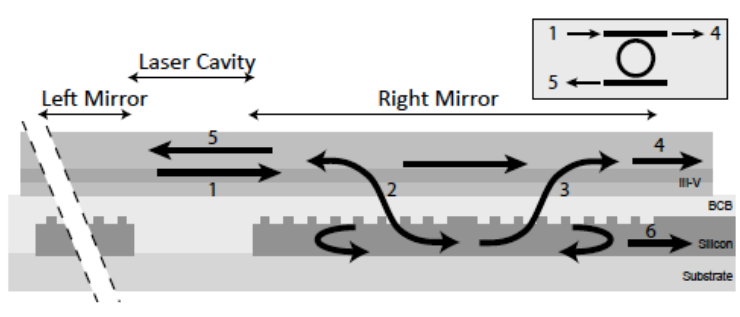

(a)

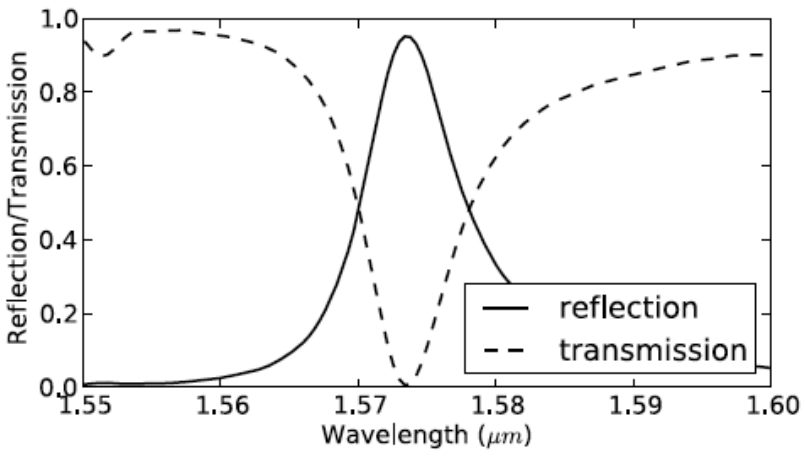

(b)

Figure 6: (a) Schematic of the resonant grating microlaser structure; (b) Simulated reflection spectrum of such a III-V silicon resonant grating device

\section{REFERENCES}

[1] F. Van Laere, G. Roelkens, M. Ayre, J. Schrauwen, D. Taillaert, D. Van Thourhout, T. F. Krauss, and R. Baets, "Compact and highly efficient grating couplers between optical fiber and nanophotonic waveguides,” J. Lightw. Technol., vol. 25, no. 1, pp. 151-156, Jan. 2007.

[2] S. K. Selvaraja, D. Vermeulen, M. Schaekers, E. Sleeckx, W. Bogaerts, G. Roelkens, P. Dumon, D. Van Thourhout, and R. Baets, "Highly efficient grating coupler between optical fiber and silicon photonic circuit," presented at Lasers and Electro-Optics/International Quantum Electronics Conference, Munich, Germany, 2009.

[3] G. Roelkens, D. Van Thourhout, and R. Baets, "High efficiency silicon-on-insulator grating coupler based on a poly-silicon overlay," Opt. Exp., vol. 14, pp. 11622-11630, 2006.

[4] D. Vermeulen, S. Selvaraja, P. Verheyen, G. Lepage, W. Bogaerts, P. Absil, D. Van Thourhout, G. Roelkens, high-efficiency fiber-to-chip grating couplers realized using an advanced CMOS-compatible silicon-on-insulator platform,Optics Express, 18(17), p.18278-18283 (2010)

[5] G. Roelkens, D. Van Thourhout, and R. Baets, "Silicon-on-insulator ultra-compact duplexer based on a diffractive grating structure," Opt. Exp. vol. 15, no. 16, pp. 10091-10096, 2007.

[6] D. Taillaert, H. Chong, P. Borel, L. Frandsen, R.M. De La Rue, R. Baets, A compact two-dimensional grating coupler used as a polarization splitter,IEEE Photonics Technology Letters, 15(9), p.1249-1251 (2003)

[7] D. Vermeulen, S. Selvaraja, P. Verheyen, P. Absil, W. Bogaerts, D. Van Thourhout, G. Roelkens, Silicon-on-insulator polarization rotator based on a symmetry breaking silicon overlay,IEEE Photonics Technology Letters, 24(6), p.482-484 (2012)

[8] R. Halir, D. Vermeulen, and G. Roelkens, "Reducing polarization dependent loss of silicon-on-insulator fiber to chip grating couplers," IEEE Photon. Technol. Letters, vol. 22, no. 6, pp. 389-391, Mar. 2010.

[9] F. Van Laere, T. Claes, J. Schrauwen, S. Scheerlinck, W. Bogaerts, D. Taillaert, L. O'Faolain, D. Van Thourhout, and R. Baets, "Compact focusing grating couplers for silicon-on-insulator integrated circuits," Photon. Technol. Letters, vol. 19, no. 23, pp. 1919-1921, 2007.

[10] F. Van Laere, W. Bogaerts, P. Dumon, G. Roelkens, D. Van Thourhout, and R. Baets, "Focusing polarization diversity grating couplers in siliconon-insulator," J. Lightw. Technol., vol. 27, no. 5, pp. 612-618, Mar. 2009.

[11] D. Vermeulen, Y. De Koninck, Y. Li, W. Bogaerts, R. Baets, G. Roelkens, Reflectionless grating couplers for SOI photonic integrated circuits ,Optics Express, p.22278-22283 (2012)

[12] Y. Li, D. Vermeulen, Y. De Koninck, G. Yurtsever, G. Roelkens, R. Baets, Compact grating couplers on silicon-on-insulator with reduced backreflection, Optics Letters, 37(21), p.4356-4358 (2012)

[13] N. Hattasan, B. Kuyken, F. Leo, E.M.P. Ryckeboer, D. Vermeulen, G. Roelkens, High-efficiency SOI fiber-to-chip grating couplers and low-loss waveguides for the short-wave infrared,IEEE Photonics Technology Letters, 24(17), p.1536-1538 (2012)

[14] A. Gassenq, N. Hattasan, E.M.P. Ryckeboer, J.B. Rodriguez, L. Cerutti, E. Tournié, G. Roelkens, Study of evanescently-coupled and gratingassisted GaInAsSb photodiodes integrated on a silicon photonic chip,Optics Express, 20(11), p.11665-11672 (2012)

[15] S. Keyvaninia, G. Roelkens, D. Van Thourhout, Christophe Jany, Marco Lamponi, Alban Le Liepvre, Francois Lelarge , Dalila Make, GuangHua Duan, Damien Bordel, Jean-Marc Fedeli, Demonstration of a heterogeneously integrated III-V/SOI single wavelength tunable laser , accepted for publication in Optics Express

[16] Y. De Koninck, F. Raineri, A. Bazin, R. Raj, G. Roelkens, R. Baets, Demonstration of a novel single-mode hybrid silicon microlaser,IEEE Photonics Conference, United States, (2012) 